\title{
EL PAPEL DE LAS PLATAFORMAS DE DISTRIBUCIÓN Y VENTA EN LA PROMOCIÓN DEL LIBRO ELECTRÓNICO INFANTIL Y JUVENIL
}

\author{
Araceli García Rodríguez* \\ Facultad de Traducción y Documentación. Universidad de Salamanca.
}

\begin{abstract}
Resumen: Partiendo de la idea del potencial que las plataformas de distribución y venta de libros electrónicos tienen para la difusión de los títulos, este artículo analiza las características que las webs de estos canales deben cumplir para potenciar la visibilidad de la literatura infantil y juvenil en torno a la oferta, las opciones de búsqueda, las posibilidades de compra, la visualización de los títulos, los sistemas de recomendación y el uso de las redes sociales. A través del análisis de las 22 plataformas más conocidas y utilizadas, se analiza igualmente en qué medida estas se adaptan a las peculiaridades propias de los consumidores de este tipo de literatura. Palabras clave: libros electrónicos; literatura infantil y juvenil; plataformas de distribución de libros electrónicos; visibilidad de la literatura infantil y juvenil.
\end{abstract}

Title: THE ROLE OF THE PLATFORMS OF DISTRIBUTION AND SALE IN THE PROMOTION OF CHILD AND YOUTH ELECTRONIC BOOK.

Abstract: Based on the idea of the potential of platforms of distribution and sale of electronic books for the dissemination of titles, this article analyzes the characteristics that the websites of these channels must meet to enhance the visibility of children's and youth literature in lathe a: bid, search options, the possibilities of purchase, display of titles recommendation systems and the use of social networks. Through the analysis of the 22 most known and used E-books platforms also analyzes to what extent these are adapted to the peculiarities of the consumers of this kind of literature.

Keywords: e-books; children's and youth literature; e-books distribution platforms; visibility of children's and youth literature.

\section{INTRODUCCIÓN.}

En el año 2000 un grupo de especialistas firmaron el "Manifiesto contra la invisibilidad de la literatura infantil y juvenil" (MANIFIESTO, 2000). Han pasado doce años y afortunadamente muchas cosas han cambiado, en lo que a los libros impresos se refiere, pero esta invisibilidad sigue siendo un condicionante que está afectando actualmente a la edición digital para niños y jóvenes.

*araceli@usal.es

Recibido: 21-1-2013 ; 2 $2^{\mathrm{a}}$ versión: 28-2-2013 ; aceptado: 4-3-2013.

GARCÍA RODRÍGUEZ, A. El papel de las plataformas de distribución y venta en la promoción del libro electrónico infantil y juvenil. Anales de Documentación, 2013, vol. 16, $\mathrm{n}^{\mathrm{o}} 1$. Disponible en: http://dx.doi.org/10.6018/analesdoc.16.1.166601 
Si partimos del concepto de visibilidad como "la capacidad de una obra para que por distintos medios llegue al conocimiento de un público potencial” (Cordón, 2004, p. 131), para que un libro, en este caso infantil y juvenil y además electrónico, tenga la posibilidad de ser conocido, vendido e incluso ser un éxito, lo primero, por supuesto, es, o debería ser, una buena historia, unas buenas ilustraciones e incluso un buen título. A partir de ahí, intervienen factores externos como la opinión de la crítica, su recomendación en una guía de lectura, la concesión de un premio, pero también, su mayor o menor presencia en los puntos de venta y por supuesto en las redes sociales.

En el caso de la Literatura Infantil y Juvenil (en adelante LIJ) impresa hace años que se realizan guías de lectura, reseñas, que se otorgan galardones, que las bibliotecas infantiles destinan una parte importante de los presupuestos a la adquisición de este tipo de obras, que las editoriales promocionan sus títulos a través de encuentros con autores y presentaciones de libros y que cuidan con mimo sus catálogos y páginas web. No parece que esté ocurriendo lo mismo en el caso de los libros digitales. Son pocas las recomendaciones hechas por especialistas; en las revistas profesionales la información sobre este formato es prácticamente inexistente. Los escasos premios que se otorgan están, por decirlo de alguna manera, en estado embrionario y son desconocidos por el público en general. Es precisamente en este contexto de invisibilidad, en el que las plataformas de distribución y venta de libros juegan un papel fundamental como herramienta para subsanar estas carencias.

"Las plataformas de comercialización de libros electrónicos son las encargadas de ofrecer al usuario y futuro cliente un amplio abanico de material en formato digital, de presentarlo de forma conveniente y atractiva a fin de que el lector cuente con todos los datos necesarios para decidir realizar la adquisición del ebook que está consultando...Además, deben ofrecerle una serie de servicios que posibiliten facilitar el proceso de adquisición a la vez que difunden y comparten información sobre su fondo bibliográfico electrónico...” (Borrel, 2012, p. 107).

Según el Informe sobre el Sector del Libro en España, el canal por excelencia para la venta de ebooks son las plataformas de distribución $(80,2 \%)$ y entre ellas, el $78,3 \%$ corresponde a las plataformas genéricas. Por ello, hay que aprovechar este potencial y conseguir, desde estos espacios, que los contenidos digitales infantiles y juveniles superen este hándicap de la invisibilidad.

Partiendo de esta premisa, es necesario plantearse dos interrogantes: ¿cómo pueden llevar a cabo esta misión? y ¿qué están haciendo al respecto? Estas dos preguntas son el punto de partida de este artículo, en el que se definen, por un lado, aquellas características que sus sitios webs, como pilar sobre el que se sustenta la visibilidad de la oferta editorial, deberían tener para hacer más visible la LIJ y, en segundo lugar, comprobar en qué medida se cumplen y cuáles de ellas se adaptan mejor a las peculiaridades propias del subsector. 


\section{METODOLOGÍA.}

Con el propósito de dar respuesta a las dos cuestiones anteriores, abordamos el análisis de las webs de diferentes plataformas que distribuyen y comercializan libros en castellano (independientemente de que dispusieran de títulos en otras lenguas). Se realizó un listado en el que se incluyeron aquellas consideradas por los especialistas como las más conocidas y utilizadas (Cordón; Gómez y Alonso, 2011, p. 261-264; INICIATIVAS, 2012) entre las cuales se seleccionaron las 22 que ofrecían un mayor catálogo infantil y juvenil con el siguiente resultado:

\begin{tabular}{|l|l|l|l|}
\hline ABACUS & CASA DEL LIBRO & GOOGLE PLAY & TIENDA DEL EBOOK \\
AMABUK & EL CORTE INGLÉS & IBOOKSTORE & TODOEBOOK \\
AMAZON & EBOOK-TEKA & LAIE & TROA \\
A. MACHADO & E-LIBRO & LEER-E & ZONAEBOOKS \\
BAJALIBROS & FNAC & LIBROSITIO & \\
BOOQUO & GRAMMATA & LUARNA & \\
\hline
\end{tabular}

Tabla I. Plataformas de distribución y venta analizadas.

Para su selección se consideró el término plataforma desde un punto de vista amplio, como sitio web en el que los clientes seleccionan y compran, independientemente de que puedan o no adquirir dispositivos de lectura, por lo que también se incluyeron librerías que comercializan este formato a través de la red. Mencionar igualmente que se excluyeron aquellas dedicadas a la distribución, pero que no permitían la compra directa, por considerar que esta era una variable interesante de cara al estudio sobre la visibilidad de la LIJ.

A continuación, se desarrolló una matriz de evaluación en la que se identificaron y seleccionaron las variables (con sus correspondientes elementos y valores), que nos permitieran conocer el comportamiento de cada plataforma y el grado de cumplimiento de los requisitos que consideramos fundamentales en lo que a la promoción de los libros para niños y jóvenes se refiere. La aplicación de la matriz mostrada en la Tabla II durante los meses de octubre y noviembre de 2012, a las 22 plataformas seleccionadas, permitió obtener datos y extraer una serie de conclusiones que se exponen a continuación.

\section{LA OFERTA.}

Un catálogo amplio en cantidad e interesante y original en cuanto a los contenidos, es un requisito esencial para esta deseada visibilidad. Es cierto que la oferta de contenidos digitales infantiles y juveniles avanza con considerable retraso a la edición en general, y que, según los datos del último Informe sobre el Sector del Libro en España, la edición digital solo supone el 3,6\% dentro del subsector y el 2,5\% sobre el total de los libros electrónicos, pero también lo es, que la presencia de los títulos en las plataformas es mínima si la comparamos con el resto de categorías. 


\begin{tabular}{|c|c|c|}
\hline VARIABLE & ELEMENTOS DE ANÁLISIS & VALOR \\
\hline Oferta & $\begin{array}{l}\text { - Oferta total } \\
\text { - Sección infantil/juvenil } \\
\text { - Sección infantil } \\
\text { - Sección juvenil }\end{array}$ & $\begin{array}{l}\quad \mathrm{N}^{\circ} \text { de títulos. } \\
\text { SI/NO y n }{ }^{\circ} \text { de títulos. } \\
\text { SI/NO y n }{ }^{\circ} \text { de títulos. } \\
\text { SI/NO y n }{ }^{\circ} \text { de títulos. }\end{array}$ \\
\hline $\begin{array}{l}\text { Opciones de } \\
\text { búsqueda }\end{array}$ & $\begin{array}{l}\text { - Materia } \\
\text { - Género } \\
\text { - Edad } \\
\text { - Colección } \\
\text { - Browsing de materias }\end{array}$ & SI/NO. \\
\hline $\begin{array}{l}\text { Opciones de } \\
\text { compra }\end{array}$ & $\begin{array}{l}\text { - Compra directa. } \\
\text { - Permite regalar. } \\
\text { - Permite descarga de aplicaciones de lectura. } \\
\text { - Sistemas de pago: } \\
\text { Tarjeta de crédito. } \\
\text { Tarjetas prepago. } \\
\text { Bonos regalo. } \\
\text { Suscripción. }\end{array}$ & $\mathrm{SI} / \mathrm{NO}$ \\
\hline $\begin{array}{l}\text { Opciones de } \\
\text { visualización }\end{array}$ & $\begin{array}{l}\text { Vista de fragmentos } \\
\text { Imagen de cubierta }\end{array}$ & $\mathrm{SI} / \mathrm{NO}$ \\
\hline $\begin{array}{l}\text { Sistemas de } \\
\text { recomendación }\end{array}$ & $\begin{array}{l}\text { - Específicas para LIJ. } \\
\text { - Tipos de recomendaciones: } \\
\text { Novedades. } \\
\text { Más vendidos. } \\
\text { Más valorados. } \\
\text { Más valorados. } \\
\text { Otras obras del autor. } \\
\text { Próximamente. } \\
\text { Títulos relacionados. } \\
\text { - Valoraciones/Puntuación. } \\
\text { - Número de valoraciones. } \\
\text { - Comentarios de los lectores. } \\
\text { - Resumen. }\end{array}$ & $\mathrm{SI} / \mathrm{NO}$ \\
\hline $\begin{array}{l}\text { Uso de redes } \\
\text { sociales }\end{array}$ & $\begin{array}{l}\text { - Permite comentar. } \\
\text { - Me gusta. } \\
\text { - Compartir (Facebook, Twiter, Tuenti, otras). }\end{array}$ & $\mathrm{SI} / \mathrm{NO}$ \\
\hline
\end{tabular}

Tabla II. Variables y elementos de análisis. 
El análisis de los 22 casos seleccionados, nos aporta unas cifras poco alentadoras ya que el fondo infantil y juvenil solo representa como media el 4,6\% de sus catálogos, porcentaje que se debe básicamente a la aportación de cuatro de ellas, Abacus, Booquo, Fnac y Amazon.

Desde el punto de vista de presentación de los catálogos, sería recomendable que las webs dispusieran de secciones independientes para el fondo infantil y el juvenil, pues esto supone una primera orientación bibliográfica para los posibles clientes, que se dirigirán a una u otra sección en función de su edad. El 29\% de los casos analizados cuentan con un catálogo infantil específico, el 26\% dispone también de un apartado juvenil y el $48 \%$ lo incluyen en una única categoría infantil y juvenil.

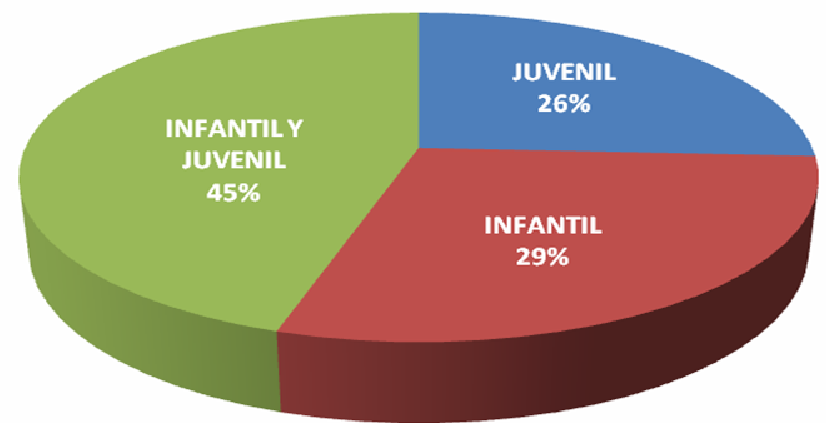

Figura 1. Distribución de los catálogos infantiles y juveniles por secciones. Fuente: Elaboración propia.

Aunque en este último caso no ha sido posible contabilizar el número de títulos destinados a niños y el destinado a jóvenes, a primera vista podemos comprobar que el mercado editorial y las plataformas, parecen decantarse más por los libros para menores de 13 años que para las etapas superiores, pese a que como han demostrado algunos estudios sobre lectura, (Carroto, 2011) este grupo es el más reacio a la lectura digital y por lo tanto deberían ser mayores los esfuerzos para publicar, promocionar y vender contenidos interesantes para este segmento.

\section{LAS OPCIONES DE BÚSQUEDA.}

Las diferentes posibilidades de búsqueda de una web pueden facilitar o dificultar la localización y visibilidad de los títulos. Una página intuitiva, en cuanto a la localización de las obras se refiere, sencilla, con una organización lógica de las diferentes categorías, puede ser un aliado para la selección, promoción y venta.

En el caso de los ebooks infantiles y juveniles, el principal requisito sería que se permitiera la búsqueda por los criterios más utilizados por los potenciales compradores de 
este tipo de literatura, niños, jóvenes y por supuesto adultos; materia, edad recomendada y la colección.

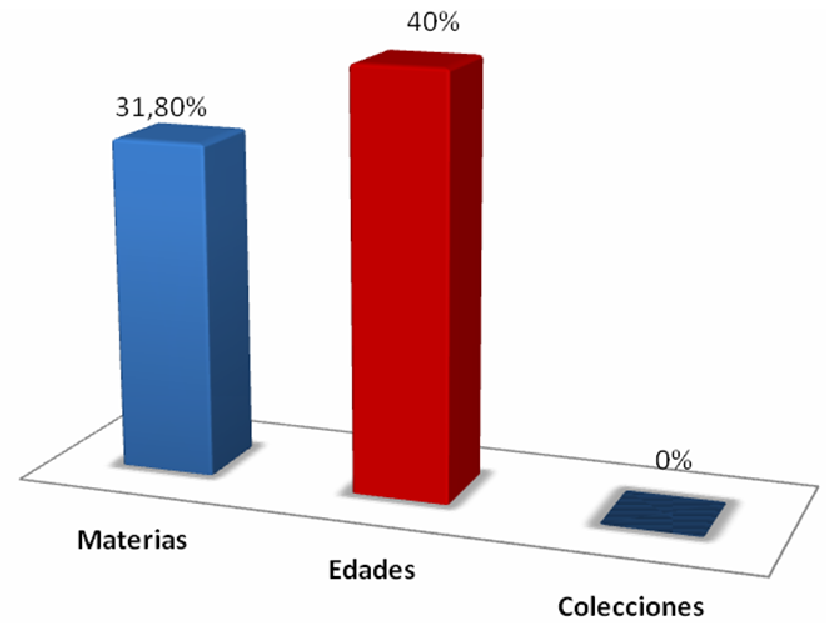

Figura 2. Porcentaje de los principales criterios de búsqueda. Fuente: Elaboración propia.

Según la Encuesta de Hábitos de Lectura y Compra de Libros del 2011, la temática es el criterio que más se tiene en cuenta a la hora de elegir un libro en la población mayor de 14 años.

Este porcentaje aumenta a medida que se va reduciendo la edad de los lectores. Por ello, esta opción de búsqueda debería estar siempre presente en todas las plataformas, preferiblemente a través de un browsing de materias específicas aunque, como hemos podido comprobar, esto ocurre en contadas ocasiones.

Solo el $31,8 \%$ de los canales, nos permiten una auténtica búsqueda por materias que además, en algunos casos, se puede combinar con edades, géneros, precios, valoraciones, etc. La utilización de criterios bibliotecarios a la hora de etiquetar y asignar materias e incluso la utilización de alguna de las clasificaciones ya aplicadas, podrían ser algunas de las soluciones a este problema.

Respecto a este apartado se pueden extraer dos conclusiones; en primer lugar la confusión terminológica, dado que la mayoría de ellas incluyen como materia el término infantil/juvenil, cuando en realidad se trata de una categoría, dentro de la cual, deberían aparecer como temas, terror, misterio, aventuras, realismo, etc; y en segundo lugar la dificultad añadida de que en ocasiones sea necesario utilizar la pestaña "Búsqueda Avanzada" para poder introducir la categoría infantil o juvenil como criterio, algo que niños y jóvenes pocas veces utilizan. 
Este público necesita tener toda la información a la vista y de forma inmediata, por lo que, como ya hemos mencionado, sería recomendable que las búsquedas pudieran realizarlas a través de browsings de materias como los que nos ofrecen Grammata o Amazon.

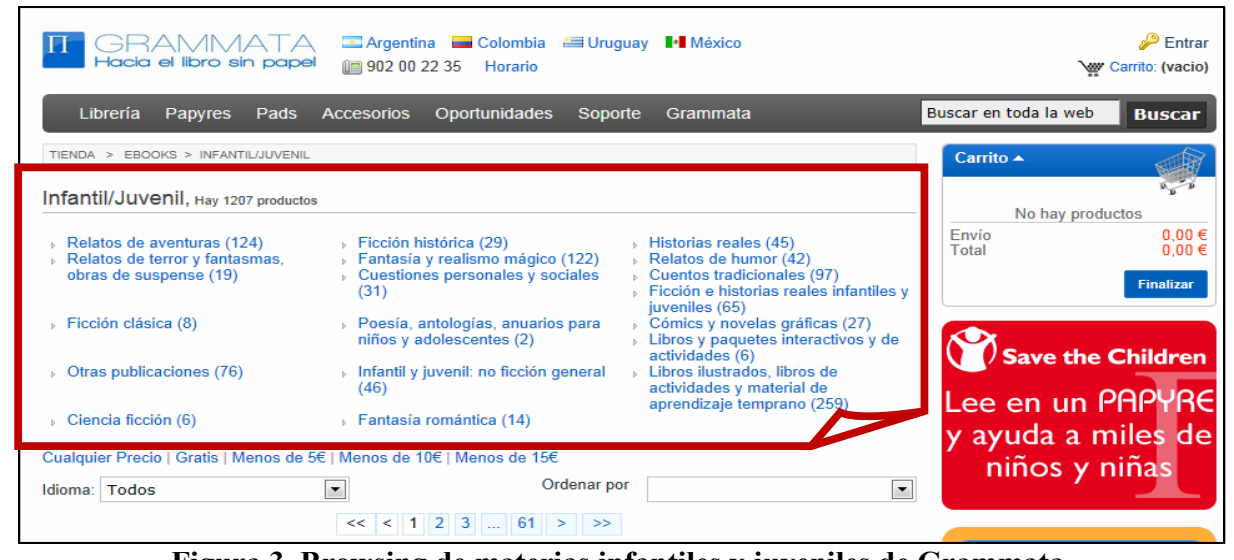

Figura 3. Browsing de materias infantiles y juveniles de Grammata.

Una buena web, debería igualmente permitir la búsqueda por grupos de edad. Es cierto que este es un criterio discutible, un mismo título puede ser válido para diferentes etapas, pero no podemos olvidarnos de que los potenciales compradores no son solo niños y jóvenes, sino también padres y que, nos guste o no, este es un criterio que utilizan habitualmente para la compra.

El que una plataforma permita limitar la búsqueda por este concepto, ayudará a los padres a seleccionar y comprar libros para sus hijos; si esta es una dinámica habitual en la literatura impresa, ¿por qué no se hace lo mismo para la edición digital?

De las plataformas analizadas, el $40 \%$ ofrecen esta posibilidad, aunque de nuevo se observa una falta total de uniformidad en cuanto a la delimitación de cada una de las etapas y una cierta confusión al incluir la edad como materia.

A esto hay que añadir el hecho de que este dato no aparezca en la descripción de los títulos, pese a que sea posible la búsqueda, y viceversa, que se haga constar la edad de los posibles lectores en la descripción, pero no se pueda localizar los títulos a través de ese campo. 


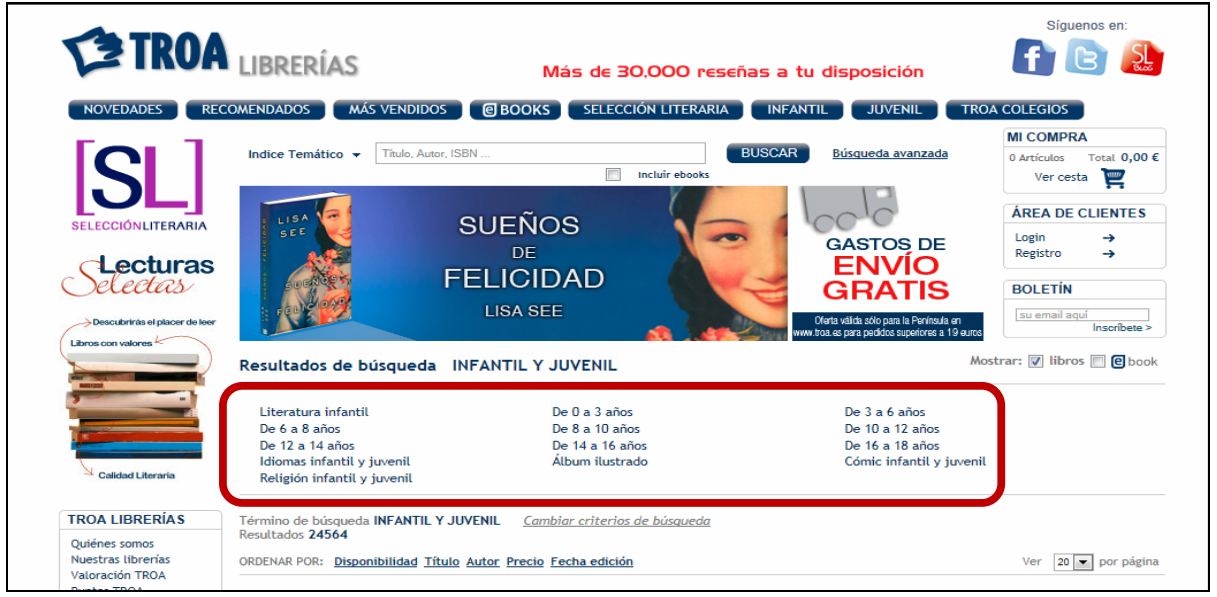

Figura 4. Búsqueda de edades en Troa.

Las colecciones son una de las señas de identidad más significativas de la edición infantil y juvenil. Una colección es una marca propia, identificable y fácilmente reconocible en cuanto a su diseño y es habitual que los niños y los jóvenes reconozcan más el nombre de una colección o de una serie que el del propio autor. Las plataformas de venta y distribución no deberían olvidarlo, dando la posibilidad a los potenciales clientes de optar por este criterio a la hora de hacer sus búsquedas. Ninguna de las webs analizadas permite localizar los títulos directamente por el nombre de la colección y salvo en dos ocasiones, Todoebook y Grammata, ni siquiera aparece el dato en la descripción.

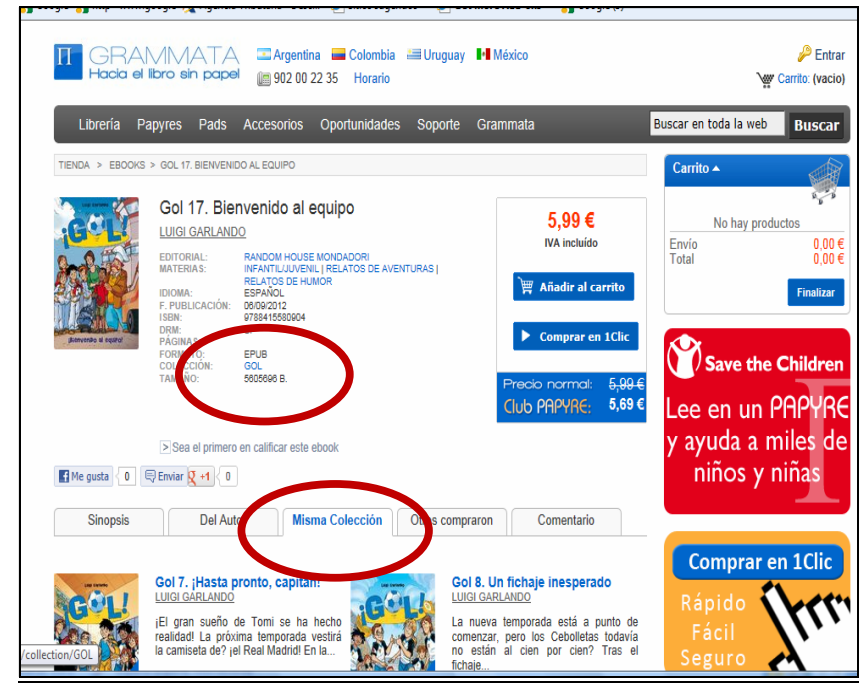

Figura 5. Búsqueda por colecciones en Grammata. 


\section{OPCIONES DE VISUALIZACIÓN.}

Las diferentes posibilidades de visualización de las obras que conforman el catálogo, pueden igualmente favorecer la visibilidad al permitir el análisis de varios títulos sin necesidad de comprarlos y, consecuentemente, un mayor conocimiento de la oferta, es decir, posibilitará algo tan habitual en las librerías en general y en las infantiles en particular, como es el ojeo/hojeo de los libros. En este caso, dos requisitos serían indispensables, mostrar la imagen de cubierta y permitir la visualización de capítulos o fragmentos.

La cubierta es la primera información que tiene el lector sobre un libro, le ayuda a reconocer personajes, identificar colecciones, contextualizar el tema y es, en el caso de los más pequeños, uno de los paratextos esenciales a través del cual pueden hacerse una idea aproximada del contenido o del protagonista de la historia. Afortunadamente en este caso el $100 \%$ de las plataformas cumplen este requisito en mayor o menor medida, pues en algunas de ellas no se muestra en todos los registros.

Sin embargo, no ocurre lo mismo con la previsualización de capítulos y/o fragmentos, que sería un requisito indispensable en los libros que cuentan con abundantes ilustraciones, ya que estas son un criterio fundamental a la hora de seleccionar los libros infantiles. Si además, esta visión de fragmentos, se mostrara a través de booktrailers (cuya elaboración lógicamente corresponde a las editoriales) que permitieran comprobar cómo se puede interactuar, con qué elementos está enriquecido, que sonidos incluye, etc., se convertiría en un poderoso sistema de atracción para niños y padres y contribuiría a romper las reticencias que estos puedan tener respecto a la lectura digital. Amabook es la única plataforma que teóricamente ofrece esta opción si bien, en la práctica, y tras consultar todos los títulos del catálogo, ninguno de los registros lleva incorporados vídeos promocionales.

De las plataformas analizadas Booquo (Echale un vistazo) y Google Play (Vista previa) permiten la visualización de las cubiertas y páginas preliminares, Ibookstore ofrece fragmentos de todos los títulos del catálogo y además posibilita la descarga de demos (Conseguir muestra), mientras que Amazon (Pruébalo gratis) proporciona la lectura gratuita del primer capítulo, aunque es necesario descargarlo de antemano, algo que sin duda desanimará a los potenciales compradores. Igualmente Grammata (Primer capítulo gratis) y el Corte Inglés (Todo view) dan la posibilidad de ver algunos de sus libros pero no la totalidad del catálogo. 


\section{OPCIONES DE COMPRA.}

Una vez seleccionado el título, las alternativas de compra también pueden influir en una mayor o menor difusión de los ebooks a través de dos mecanismos, la opción de utilizar tarjetas pre-pago tipo i-Tunes y la de regalarlo a un simple golpe de ratón.

En el primer caso, el hecho de que los lectores dispongan de una tarjeta de estas características, puede facilitar la compra por impulso sin la necesidad de la presencia de un adulto que tenga que introducir los datos y controlar, su tarjeta de crédito. Solo dos casos, Google Play e Ibookstore admiten este sistema dentro de sus opciones de compra, si bien se recogen otras alternativas similares como es el caso de Amabook o Amazon, que permiten la adquisición de bonos regalo.

Igualmente algunas plataformas proporcionan la descarga gratuita de aplicaciones de lectura para el Ipad e Iphone, lo que subsana en buena medida este inconveniente, al poder hacer la compra a través de este dispositivo utilizando la tarjeta i-Tunes.

Otra fórmula interesante y de grandes posibilidades para los niños y jóvenes, es el sistema Premium de Booquo que permite, mediante la suscripción, el acceso ilimitado a la biblioteca digital y a diferentes canales temáticos entre los cuales aparece, Booquoland, un espacio para niños y jóvenes en el que se incluyen novedades, obras más vendidas y más valoradas, una alternativa interesante para promocionar la LIJ dentro de la plataforma y consecuentemente para hacerla más visible.

Por lo que se refiere a la posibilidad de regalo, el hecho de que en cada uno de los registros esté destacada la opción "Regalar", puede ayudar a que los clientes obsequien ebooks igual que lo harían con un libro en papel. No podemos olvidarnos de la influencia del factor regalo en la venta de títulos infantiles y juveniles; un alto porcentaje se vende como obsequio y los canales de venta tienen que aprovechar esta oportunidad y conseguir que resulte igual de atractivo, y en este caso mucho más rápido, a golpe de clic, regalar en digital que en papel. Solo cinco de los canales analizados (Todoebook, Zonaebook, El Corte Inglés, La Casa del Libro y Laie) permiten regalar directamente, cuatro (Amazón, Amabook, Google Play e Ibookstore) dan la posibilidad de hacer bonos o tarjetas regalo pre-pago, mientras que en la Tienda del Ebook, aunque no es posible hacerlo desde la descripción del mismo título, ofrece esta función a través del apartado "Mis listas de regalo". 


\section{SISTEMAS DE RECOMENDACIÓN.}

A través de los diferentes canales de venta y distribución se puede dar mayor visibilidad a la LIJ incorporando orientaciones bibliográficas, listados de novedades, información sobre los títulos más y mejor valorados, avances de publicación y recomendaciones de lectura específicas, esenciales para promocionar los títulos, para servir como referente a la hora de seleccionar y para que haya un mayor conocimiento sobre ellos.

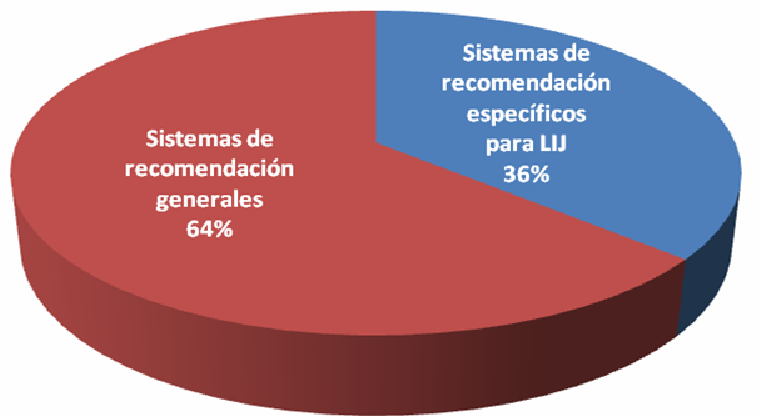

Figura 6. Sistema de recomendación de libros electrónicos. Fuente: Elaboración propia.

Aunque en todos los casos analizados se incorporan este tipo de secciones, lamentablemente solo el 36\% dispone de instrumentos de orientación específicos para los niños y jóvenes que incluyen normalmente novedades, los más vendidos y los más valorados (por los lectores), así como recomendaciones, bien a través de un browsing o marcando cada título con frases como "recomendado", "destacado", etc. 

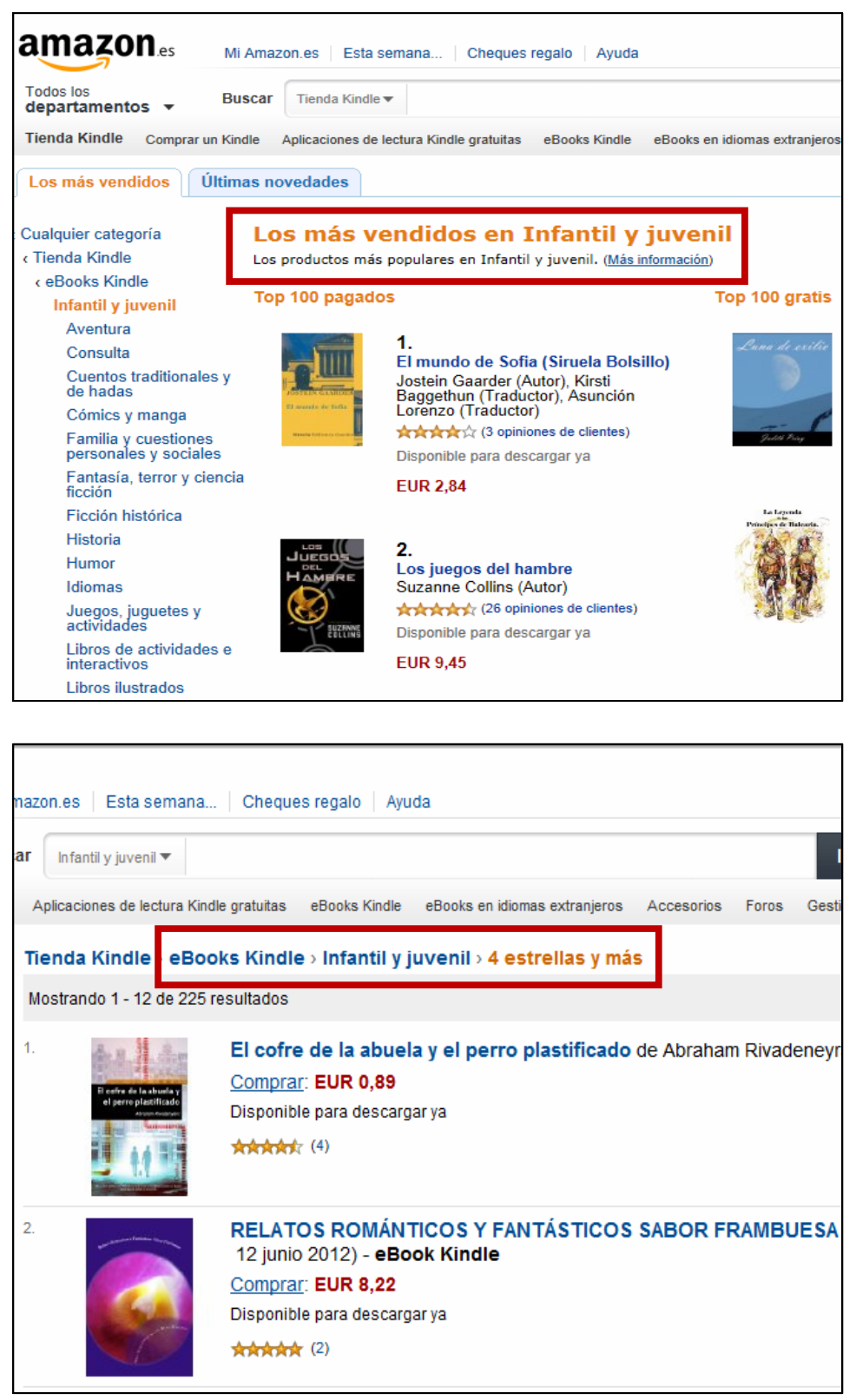

Figura 7. Sistemas de recomendación de Amazon.

Se puede considerar también una buena estrategia, en lo que a procedimientos de recomendación se refiere, dar una información lo más completa posible sobre cada uno de

Anales de Documentación, 2013, vol. 16, nº 1 
los títulos, requisito que se cumple en mayor o menor medida en todos los casos, pero además sería especialmente aconsejable:

- Incorporar en la descripción un resumen de la obra

- Incluir el número de valoraciones recibidas y la puntuación obtenida, (preferiblemente a simple vista sin necesidad de entrar en cada uno de los títulos y a través de iconos)

- Ofrecer información biográfica sobre el autor

- Mostrar otros títulos del mismo autor disponibles en el catálogo

- Anunciar próximas publicaciones para generar expectación

- Indicar otras obras relacionadas para que el lector descubra títulos desconocidos, pero igualmente interesantes

En el siguiente gráfico se muestran como los diferentes canales se ajustan a los requisitos anteriores.

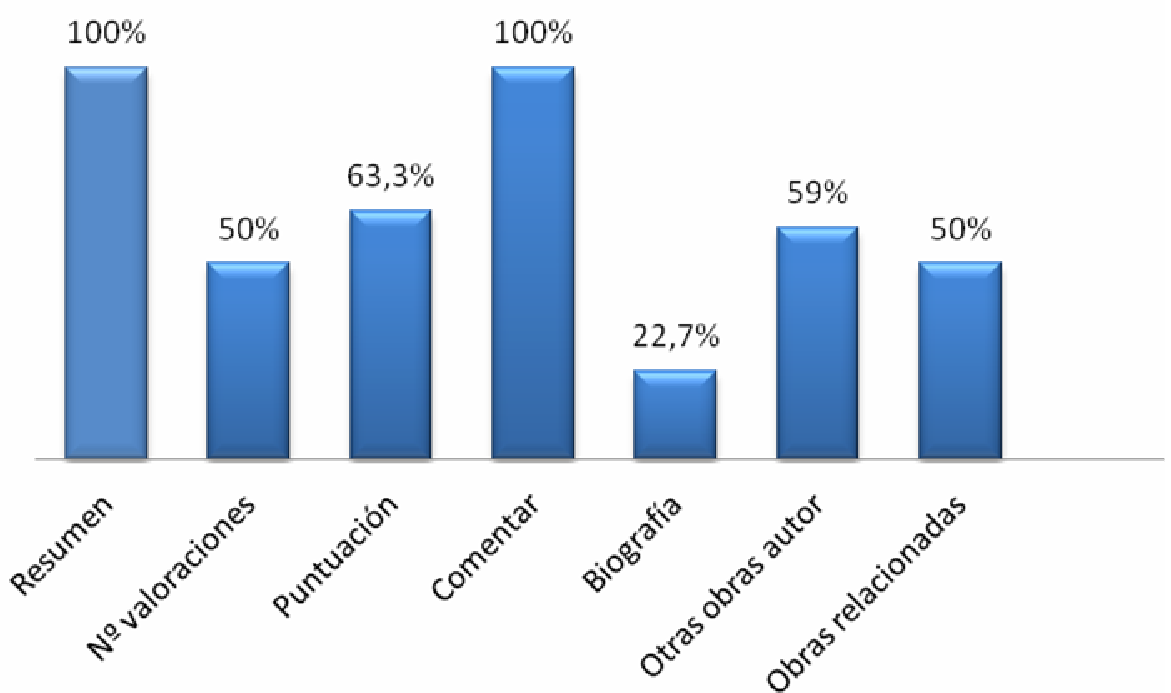

Figura 8. Procedimientos para la recomendación de títulos. Fuentes: Elaboración propia.

Por lo que se refiere al resumen, todas las plataformas lo incluyen como parte de la descripción, otra cosa es si son realmente útiles. En general son bastante simples, en ocasiones una única frase, y en el caso de existir versión en papel, simples trascripciones de los que aparecen en las contracubiertas. La única excepción que podemos hacer al respecto es la de Troa pues las valoraciones y las sinopsis de los libros, al menos así consta en su web, están realizadas por profesionales de la plataforma. 


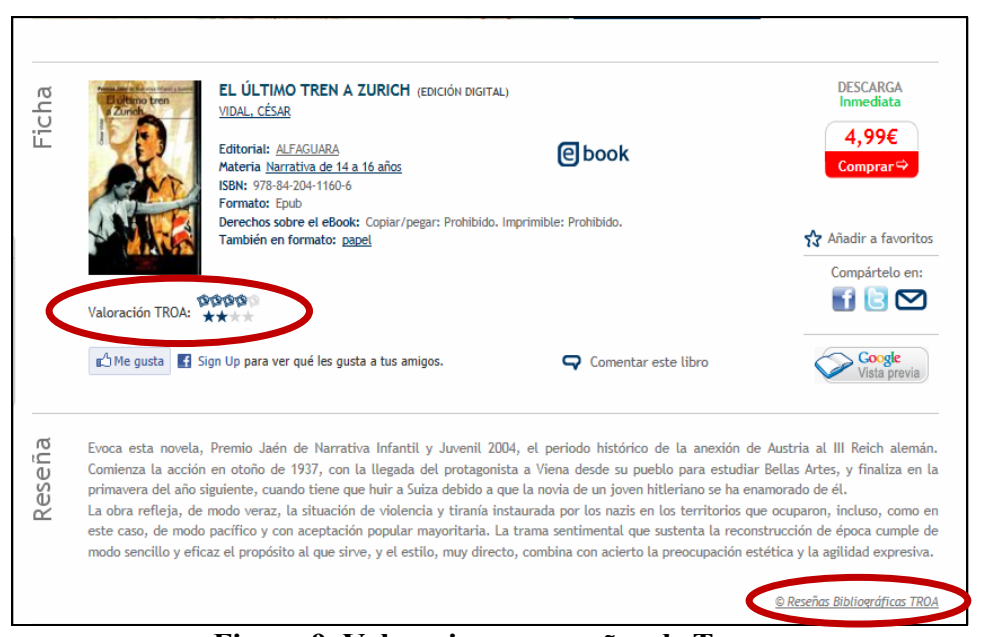

Figura 9. Valoraciones y reseñas de Troa.

El 50\% de los casos estudiados, permiten verificar el número de valoraciones recibidas y el $63,3 \%$ ofrece a los clientes la posibilidad de puntuar y comprobar la calificación obtenida, si bien, en ocasiones, esta información es poco fiable al encontrarnos con ebooks con una puntuación máxima de cinco, pero que se debe a un solo lector.

La opción Valorar/Puntuar, es especialmente útil para los más pequeños ya que redactar algún tipo de comentario, aunque sea breve, puede suponer un esfuerzo que en ocasiones no están dispuestos a realizar, pero sí les resulta cómodo manifestar su opinión con un solo clic. Por este motivo es habitual encontrarnos con obras valoradas, en las que no aparece ningún tipo de comentario adicional o en las que estos están realizados por los padres.

La opción Comentar es más utilizada por jóvenes y adultos. Todos las plataformas analizadas permiten a sus clientes comentar y visualizar los comentarios realizados por otros lectores, si bien, hay que matizar, que el numero de críticas recibidas por los títulos infantiles y juveniles son más bien escasas, entre tres y cinco por registro, y que la mayoría incluyen opiniones muy breves que dan pocas pistas sobre la validez de los diferentes títulos; son habituales frases como "engancha", "no puedes parar de leer", "aburrido", "no me ha gustado nada".. La Casa del Libro es el canal que más información nos ofrece al respecto indicando, no solo el número total de valoraciones, sino también la puntuación media recibida y el porcentaje de recomendaciones de los usuarios en función de ocho categorías como "Para regalar", "Para disfrutar", "No puedes perdértelo", etc. 


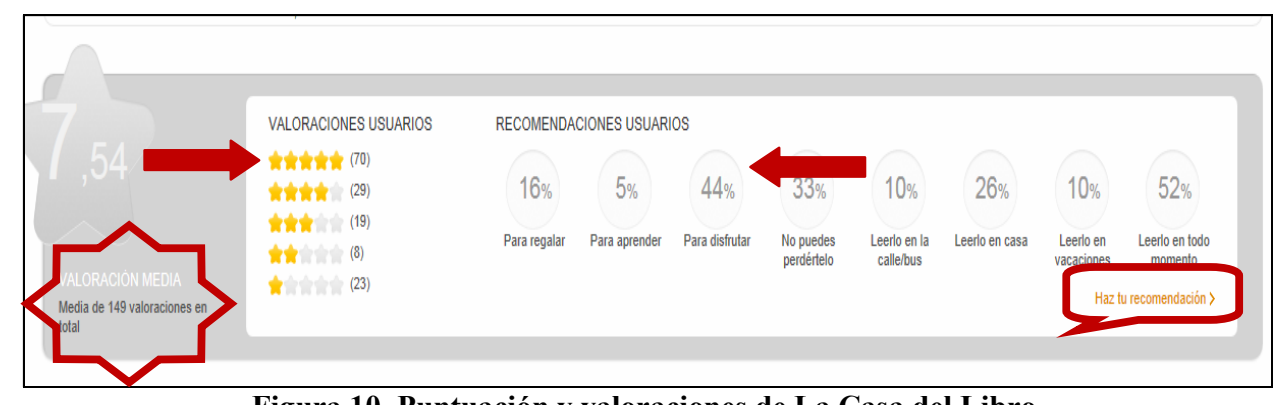

Figura 10. Puntuación y valoraciones de La Casa del Libro.

En cuanto al resto de las recomendaciones señaladas, el 22,7\% incluye información biográfica sobre el autor, normalmente muy breve, el 59\% indica otras obras del autor dentro del catálogo, el 13,6\% muestran próximos lanzamientos, mientras que el 50\% reseñan otras obras relacionadas bien por su temática, edad, u otros criterios en ocasiones un tanto complicados de definir y que pueden dar lugar a recomendaciones cuanto menos, sorprendentes.

\section{USO DE LAS REDES SOCIALES.}

Si hay un factor decisivo al que pueden contribuir los portales de distribución y venta para que la LIJ digital sea cada vez más visible, es la posibilidad de utilizar las redes sociales para incluir comentarios, opiniones, libros favoritos, etc. Niños y jóvenes demandan, cada vez más, poder compartir sus lecturas y comprobar que opinan sus semejantes sobre ellas. Además, las redes sociales pueden ayudar a crear expectación o interés por determinadas obras, al tiempo que los editores pueden conocer de primera mano las opiniones de los lectores, adecuar sus catálogos e incluso personalizarlos.

El potencial de estas herramientas para promocionar un título y para influir en su selección y compra es cada vez mayor en el libro en general y en el libro infantil y juvenil en particular. La presencia de una obra en las redes sociales aumenta su posibilidad de ser conocida, y por lo tanto de ser más visible, sin la necesidad de llevar a cabo costosas campañas de marketing.

Según la última Encuesta de Hábitos de Lectura y Compra de Libros, el 17\% de los mayores de 14 años, utilizan como criterio para la selección el consejo o recomendación de un amigo, lo que viene a corroborar lo que todos los que nos dedicamos a trabajar en el campo de la LIJ sabemos: que por mucho que los profesionales hagan recomendaciones, con los niños y los jóvenes lo que mejor funciona es el "boca a boca”. Si además tenemos en cuenta, que más del $70 \%$ de los menores internautas son usuarios habituales de las redes sociales, (Bringué; Sádaba, 2011) y que este porcentaje aumenta hasta el $91 \%$ para los mayores de 14 años, es fundamental que puedan compartir sus lecturas a través de estas redes. Por ello, aumentar la visibilidad de los títulos infantiles y juveniles pasa necesariamente por dar la posibilidad de compartir comentarios a través de las más 
utilizadas por este tipo de lectores, Tuenti para los menores de 12 años y Facebook, Twiter y Myspace, por este orden, para los mayores de 12 años.

De los 22 portales analizados el 95,4\% cuentan con un espacio en una o varias de las principales redes sociales, espacio que utilizan básicamente para promocionar las obras, dar a conocer novedades, ofrecer noticias sobre la plataforma, sobre dispositivos de lectura, etc., pero solo el $63 \%$ permite a sus usuarios compartir directamente sus opiniones desde cada uno de los títulos.

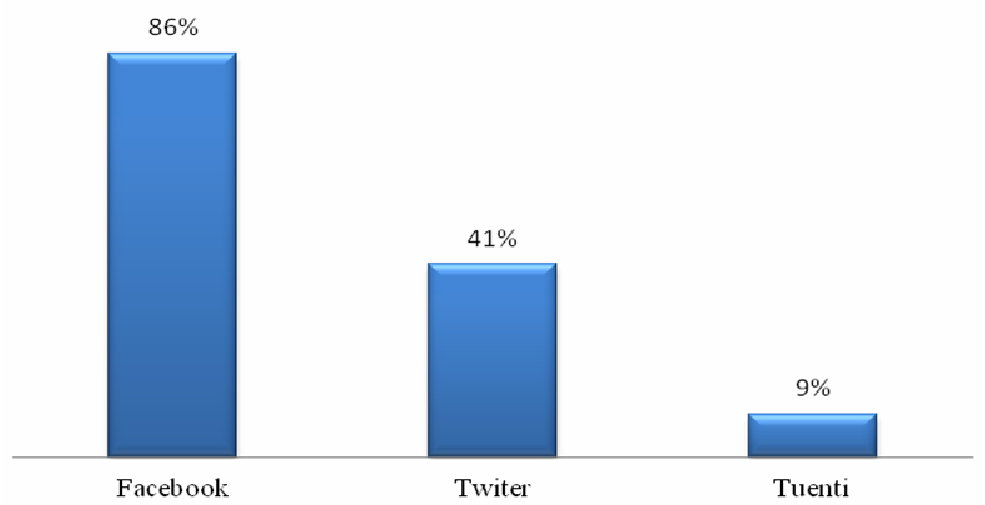

Figura 11. Redes sociales utilizadas por las plataformas. Fuente: Elaboración propia.

Facebook es la más utilizada, mientras que Twiter ocupa el segundo lugar con el 23\%, y llama poderosamente la atención que solo el $6 \%$ interactúen con sus lectores infantiles y juveniles a través de Tuenti, más cuando, como hemos mencionado, es la más manejada entre los menores de 12 años. En este sentido las plataformas que nos ofrecen un mayor número de opciones son Ebook-teca y Amabook, que permiten compartir en más de 300 redes sociales de todo el mundo.

\section{CONCLUSIONES.}

El sector digital infantil y juvenil tiene en las plataformas generalistas una forma de darse a conocer, un escaparate en el que promocionarse y hacerse más visible. Para la consecución de este objetivo, a la espera de que se vayan consolidando proyectos de plataformas especializadas, sus espacios webs deben adaptarse a las características propias del género y modificar sus estrategias en torno a oferta, opciones de búsqueda, posibilidades de compra, visualización de títulos, sistemas de recomendación y uso de las redes sociales.

Por lo que se refiere a la oferta, la presencia de la LIJ es mínima frente a los miles de títulos disponibles para adultos. Es cierto que según los datos de las estadísticas oficiales, 
la oferta es más reducida, pero también que estos canales no se preocupan excesivamente por este género en cuanto al volumen de títulos ofertados.

Los gustos, aficiones o intereses no son los mismos para los niños que para los jóvenes. Sin embargo, la mayoría de las web incluyen todos los títulos en una categoría conjunta infantil y juvenil, un aspecto que debería mejorarse potenciando, en la medida de lo posible, los libros para los jóvenes, que según han demostrado diferentes estudios, son los más reacios a la lectura digital y por lo tanto los que más necesitan conocer las posibilidades de un ebook.

En cuanto a las opciones de búsqueda, son varias las líneas de mejora que deberían plantearse de cara una mayor visibilidad:

- Diseñar browsings de materias adaptados a los gustos e intereses de estos clientes, con unos términos claros en los que no se produzca confusión entre temas, géneros o edades

- Favorecer la localización de los títulos por parte de los adultos incluyendo como criterio de búsqueda las etapas lectoras

- Ofrecer datos sobre la colección y permitir su localización a partir de este campo

La posibilidad de visualización de fragmentos y/o capítulos, o la incorporación de booktrailers, una de las asignaturas pendientes de la mayoría de las plataformas, puede ser una herramienta esencial para dotar a la LIJ de una mayor visibilidad, al permitir que los clientes examinen los libros como si estuvieran en una librería tradicional.

Aunque todavía de forma minoritaria, se están desarrollando sistemas que faciliten la compra por impulso y potencien el factor regalo. Son mecanismos que ayudarán a un mayor conocimiento de los ebooks infantiles, como es el caso de las tarjetas pre-pago, los bonos regalo, la suscripción y la posibilidad de regalar a un solo golpe de clic.

Cada vez más, los consumidores de este tipo de literatura, demandan puntos de referencia que les permitan encontrar y seleccionar aquellos títulos más interesantes, por lo que es necesario que se desarrollen instrumentos de orientación bibliográfica específicos para el género. Se deben potenciar las recomendaciones de los propios lectores dándoles la posibilidad de valorar/puntuar sus lecturas sin necesidad de incluir ningún tipo de comentario adicional, que en la mayoría de los casos, tienen muy poca utilidad.

Las redes sociales representan una muy buena opción para conseguir la visibilidad de la LIJ, permiten compartir lecturas, conocer las opiniones de sus semejantes e incluso pueden crear expectación sobre determinados títulos. Las plataformas de venta y distribución deben ser conscientes de este potencial y ofrecer la posibilidad de compartir sus comentarios directamente desde cada uno de los registros del catálogo 
A modo de conclusión general, podríamos decir que la plataforma ideal, en lo que a LIJ se refiere, estaría formada por una combinación del fondo bibliográfico de Amazon, Google Play e Ibookstore; las opciones de búsqueda de la Casa del Libro, Troa y Amazón; las posibilidades de compra de Ibookstore, Google Play, Booquo y Amazon; los sistemas de recomendación de Troa, Booquo y la Casa del Libro y el potencial de uso de las redes sociales de Eboo-Teka y Amabook.

\section{BIBLIOGRAFÍA.}

BRINGUÉ, X. y SÁDABA, C. Menores y redes sociales en España. Madrid: Foro Generaciones Interactivas, 2011.

BORREL, M. Plataformas de comercialización de libros electrónicos. En: CORDÓN, J.A.; CARBAJO, F.; GÓMEZ, R. y ALONSO, J. (coords). Libros electrónicos y contenidos digitales en la sociedad del conocimiento: mercados, servicios y derechos. Madrid: Pirámide, 2012, p. 105-128.

CARROTO, P. La generación digital que ama el papel [en línea]. Disponible en: $<$ http://www.publico.es/culturas/365547/la-generacion-digital-que-ama-el-papel $>$. [Consulta: 21 de octubre de 2012].

CORDÓN, J.A. La visibilidad en los circuitos de la creación literaria: literatura y traducción. En: MANUAL de documentación y terminología para la traducción especializada. Madrid: Arco Libros, 2004, p. 127-169.

CORDÓN, J.A.; GÓMEZ, R. y ALONSO, J. Gutenberg 2.0: la revolución de los libros electrónicos. Gijón: Trea, 2011.

CORDÓN, J.A.; ALONSO, J. y GÓMEZ, R. Modelos de negocio y plataformas de venta de libros electrónicos en España [en línea]. Disponible en: $<$ http://gredos.usal.es/jspui/bitstream/10366/83511/1/cordon.pdf $>$. [Consultado: 16 de octubre de 2012].

CORDÓN, J.A.; ALONSO, J. y DÍAZ, R. Las plataformas de venta de libros electrónicos: modelos de negocio y estrategias de mercado [en línea] Disponible en: $<$ http://www.ub.edu/bid/26/cordon2.htm $>$. [Consultado: 26 de octubre de 2012].

ESCRIBANO, A. La promoción del libro electrónico. En: CORDÓN, J.A.; CARBAJO, F.; GÓMEZ, R. y ALONSO, J. (coords). Libros electrónicos y contenidos digitales en la sociedad del conocimiento: mercados, servicios y derechos. Madrid: Pirámide, 2012, p. 159-179.

HÁBITOS de lectura y compra de libros en España 2011 [en línea]. Disponible en: $<$ http://www.federacioneditores.org/0_Resources/Documentos/Habitos_lectura_Comp raLibros_2011.pdf>. [Consultado: 22 de octubre de 2012].

INFORME sobre el sector del libro en España: 2010-2012 [en línea]. Disponible en: $<$ http://www.mcu.es/principal/docs/MC/2012/Libro/Sector_del_Libro_Abril_2012.pdf $>$.

[Consultado: 13 de octubre de 2012].

INICIATIVAS Editoriales y Librerías de Recursos Web. Observatorio de la Lectura y el Libro [en línea]. Disponible en:

$<$ http://www.mcu.es/libro/MC/ObservatorioLect/RecursosWeb/Iniciativas.html>.

[Consultado: 3 de octubre de 2012]. 
MANIFIESTO contra la invisibilidad de la Literatura Infantil y Juvenil [en línea]. Disponible en: <http://www.imaginaria.com.ar/04/1/manifiesto.htm>. [Consultado: 3 de noviembre de 2012].

OPORTUNIDADES de distribución digital internacional para el sector editorial [en línea]. Disponible en:

$<$ http://www.icex.es/icex/cma/contentTypes/common/records/mostrarDocumento/?doc $=4564831>$. [Consultado: 2 de noviembre de 2012].

PANORÁMICA de la Edición Española de libros 2010 [en línea]. Disponible en: $<$ http://www.calameo.com/read/000075335b459ef70901e $>$. [Consultado: 21 de octubre de 2012].

EL SECTOR del libro en España: 2010-2012 [en línea]. Disponible en: $<$ http://www.mcu.es/libro/docs/MC/Observatorio/pdf/Sector_libro_201012_sep12.pdf $>$. [Consultado: 18 de noviembre de 2012]. 